Document downloaded from:

http://hdl.handle.net/10251/151083

This paper must be cited as:

Libich, J.; Perez, J.; Zvanovec, S.; Ghassemlooy, Z.; Nebuloni, R.; Capsoni, C. (2017). Combined effect of turbulence and aerosol on free-space optical links. Applied Optics. 56(2):336-341. https://doi.org/10.1364/AO.56.000336

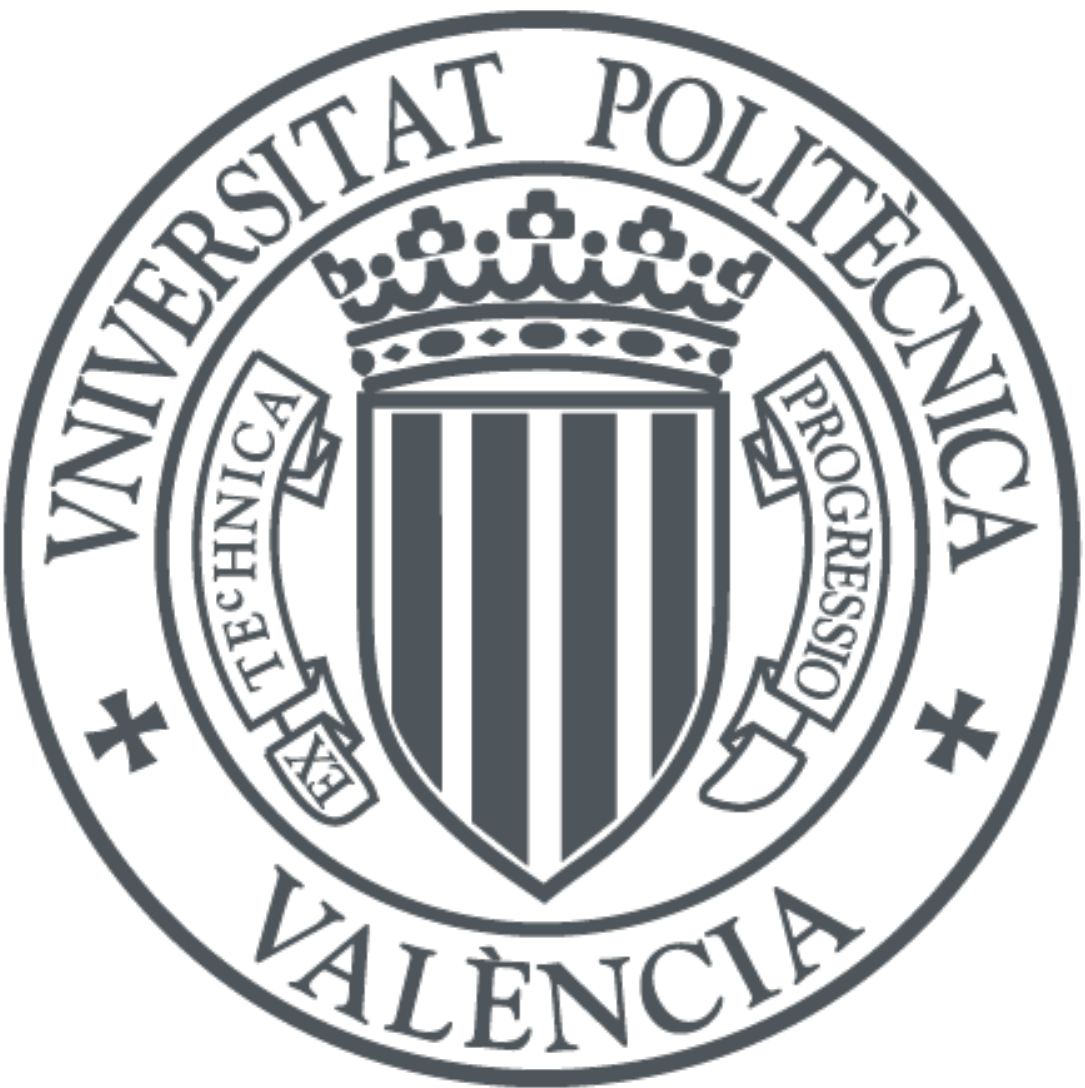

The final publication is available at

https://doi.org/10.1364/AO.56.000336

Copyright The Optical Society

Additional Information 


\title{
Combined Effect of Turbulence and Aerosol on Free Space Optical Links
}

\author{
JiRI Libich, ${ }^{1}$ Joaquin Perez, ${ }^{*}, 2$ Stanislav ZVAnovec, ${ }^{1}$ Zabih Ghassemlooy, ${ }^{3}$ \\ Roberto Nebuloni, ${ }^{4}$ and Carlo Capsoni ${ }^{5}$
}

\author{
${ }^{1}$ Faculty of Electrical engineering, Department of electromagnetic fields, Czech Technical University in Prague, Technicka 2, Prague, Czech \\ Republic \\ ${ }^{2}$ Photonics Research Labs, iTEAM Research Institute, Universitat Politècnica de València, Camino de Vera, 46022 Valencia, Spain \\ ${ }^{3}$ Optical Communications Research Group, NCRLab, Faculty of Engineering and Environment, Northumbria University, Newcastle upon Tyne, \\ NE1 8ST, UK \\ ${ }^{4}$ Istituto di Elettronica e di Ingegneria dell'Informazione e delle Telecomunicazioni, Consiglio Nazionale delle Ricerche, Milan, Italy, \\ ${ }^{5}$ Politecnico di Milano, Milan, Italy \\ *Corresponding author: joapeso@upv.es
}

Received XX Month XXXX; revised XX Month, XXXX; accepted XX Month XXXX; posted XX Month XXXX ((Doc. ID XXXXX); published XX Month XXXX

\begin{abstract}
Despite the benefits of free-space optical (FSO) communications, their full utilization is limited by the influence of atmosphere weather conditions. Such as fog, turbulence, smoke, snow and others. In urban environments, additional environmental factor such as smog and dust particles due to air pollution caused by the industry and motor vehicles may affect the FSO link performance, which has not been investigated in detail yet. Both smog and dust particles cause absorption and scattering of the propagating optical signal, thus resulting in high attenuation. This work investigates the joint impact of the atmospheric turbulence and dust particle imposed scattering on the FSO link performance as part of the last mile access network in urban areas. Propagation of an optical wave is at first analyzed based on the micro-physic approach and the extinction caused by small particles is determined. An experimental measurement campaign using a dedicated test chamber is carried out to assess the FSO link performance operating wavelengths of 670 and $830 \mathrm{~nm}$ and under the dust and turbulent conditions. The measured attenuation and the $Q$-factor in terms of the velocity of particle flow and the turbulence strength are analyzed. We show that for an air flow of $2 \mathrm{~m} / \mathrm{s}$ the $Q$-factor is almost 3.5 higher at the wavelength of $830 \mathrm{~nm}$ than at $670 \mathrm{~nm}$. However, for a wavelength of $670 \mathrm{~nm}$ the FSO link is less affected by the increase in airflow compared to $830 \mathrm{~nm}$. The $Q$-factor reduces with turbulence. Under similar turbulence conditions, for the ash particles the $Q$-factor is higher than that of sand particles. () 2016 Optical Society of America
\end{abstract}

OCIS codes: (060.2605) Free-space optical communication; (010.1330) Atmospheric turbulence; (010.1110) Aerosols.

https://doi.org/10.1364/AO.56.000336

\section{INTRODUCTION}

The growing popularity of free-space optical (FSO) links as part of optical wireless communications (OWC) for mostly outdoor applications is evident from the increasing number of publications in the last decade [1,2]. In large metropolitan cities, the demand for providing a high-speed backbone network for wireless communications [3] and last mile access networks without the need for digging the streets (i.e., the need for regulations, high cost and inconvenience) are rapidly increasing, which requires viable solutions. In such situations, FSO systems do have a role to play in providing high data rate interconnection [4] between buildings and being used as the interface between existing communication infrastructures. They are very fast to deploy and offer backbone type data rates similar to optical fiber at a relatively acceptable cost [5]. Additionally, FSO links can be readily used in situations where there is a big demand for the radio frequency (RF) spectrum, e.g., big sporting events and concerts, or in emergency situations such as flooding, fire and security alerts, etc., in order to rapidly provide high-quality and high-speed wireless communication connectivity. Moreover, OWC links based on the FSO technology are also being recognized as fundamental for the development of mobile front-haul and backhaul in urban areas, which will contribute to the fifth generation (5G) wireless communications standard as a tool to augment the capacity due to the spectrum crunch facing the RF wireless systems [3].

However, similarly to radio waves, optical waves are influenced by many factors when propagating through the atmosphere. Particles in the atmosphere scatter and or absorb light waves. The wave-particle interaction results in attenuation due to fog, snow, rain, smog, and 
turbulence [5-7]. Optical attenuation increases from a mere $0.2 \mathrm{~dB} / \mathrm{km}$ on a very clear day to about $300 \mathrm{~dB} / \mathrm{km}$ in dense fog conditions (i.e., visibility down to about $50 \mathrm{~m}$ ). A high level of air pollution (smoke, dust and smog) combined with a high level of humidity in urban areas decreases the FSO link visibility to tens of meters, thus limiting the link availability well below the expected $99.999 \%$ [8,9]. Air pollution due to smog is primarily caused by human activities such as transportation, industry, heating systems, agriculture, and forest fires [10]. Smog is composed of particulate matters (PMs) such as nitrogen oxides (NOx), sulphur dioxide $\left(\mathrm{SO}_{2}\right)$, volatile organic compounds (VOCs) and ammonia, ground-level ozone and gases formed by the reaction between NOx and VOCs in the presence of sunlight. PM10 particles (i.e. the fraction of particulates in the air of size $<10 \mu \mathrm{m}$ ) and PM2.5 particles $(<2.5 \mu \mathrm{m})$ are of major concern, as they are small enough to penetrate deep into the lungs, and so potentially pose significant health risks [5] [11].

Studies of the FSO link performance mostly with fog and to some extent sandstorms have been reported in [12-14]. However, in real scenarios, especial in the cities with hot dry weather conditions FSO links may also experience smog, and dust storms where dust particles are up-lifted up to 1-3 km into the atmosphere and can travel long distances for days [15]. Although several studies and experimental data on the FSO link performance considering either smog (mostly dust) or turbulence are available in the literature [16-18], to the best of our knowledge, no studies on the combined effect of smog and turbulence, which are rather frequent in subtropical metropoles, have been reported.

In this paper, for the first time, we experimentally investigate the effect of both turbulence and smog particles, mainly dust, on the FSO link performance under a controlled indoor environment. We assess the link performance operating at wavelengths $\lambda$ of 670 and $830 \mathrm{~nm}$ in terms of the attenuation and the $Q$-factor as a function of the velocity of particle flow and the turbulence strength.

The rest of the paper is structured as follows. The theoretical principles of wave-particle interaction and turbulence effects are briefly given in Section 2. In Section 3, the experiment setup based on a dedicated FSO dust chamber is described, whereas the results are outlined and discussed in Section 4. Finally, the conclusions are given.

\section{THEORETICAL BACKGROUND}

In order to assess the performance of the FSO communications link under smog conditions (particularly both sand and ash particles), it is necessary to characterize these particles in terms of their size and composition since they will contribute to the optical attenuation. First, the energy loss of an optical wave travelling through a layer of spherical particles with different radii can be expressed by the extinction coefficient, which is defined by [19]:

$$
\gamma=\int_{0}^{\infty} \pi r^{2} n(r) Q_{e x t}(r) d r,
$$

where $r$ is the particle radius, $n(r)$ stands for the particle size distribution and $Q_{\text {ext }}$ is the extinction efficiency factor of the particle.

Figure 1 shows the extinction efficiency factor as a function of the sand particle radius $r$ with a 5\% volume content of hematite for a range of optical wavelengths. The optical properties of the particles have been adopted from [20].

In general, the scattering particles have a complex shape, which is usually approximated by a spheroid, for electromagnetic computational efficiency. According to the spheroid aspect ratio $s / t$ (where $s$ is the equatorial radius of the spheroid, and $t$ is the distance from center to the pole along the symmetry axis) they are divided into oblate $(s / t>1)$ and prolate $(s / t<1)[21]$ and those having $s / t=1$ are classified as spheres.
The properties of spheroidal particles are usually described in terms of the diameter $D$ of an equal-volume sphere, where $D=2 \sqrt[3]{\left(s t^{2}\right)}$ [21]. For propagation through a layer of spheroids, $Q_{e x t}$ depends both on $r$ and the shape distribution as well as the particle orientation relative to the direction of the incident light. In the case of sand and ash (solid) particles, due to the large number of particles and under the turbulence condition, we have assumed that particles orientation is random. Therefore, absorption, scattering and consequently attenuation of a propagation optical beam are independent of both the polarization and the orientation. According to Paramonov approximation [22], randomly oriented spheroids are optically equivalent to polydisperse spherical particles based on a certain weight function.

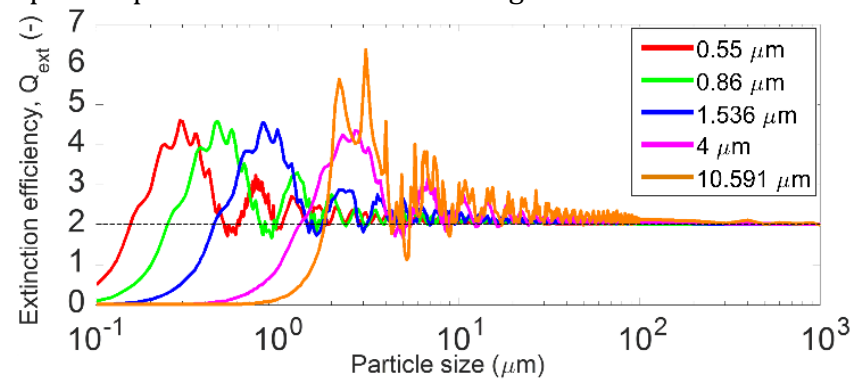

Fig. 1. Extinction efficiency factor $Q_{\text {ext }}$ against radius of sand particles assuming a $5 \%$ volume content of hematite at five different optical wavelengths.

Turbulence results in an inhomogeneous distribution of the refractive index of air, which, in turn, leads to optical beam widening and wandering. These effects result in random fluctuations of the received optical power and the phase. According to the well-established model of the weak turbulence regime, the power fluctuations are quantified by the scintillation index, which in the case of a plane wave is given by [5]:

$$
\sigma_{I}=1.23 C_{n}^{2} k^{7 / 6} L^{11 / 6},
$$

where $k$ is the wave number of the optical wave, and $L$ is the link length. $C_{n}{ }^{2}$ is the structure parameter of the refractive index of the atmosphere. It can be derived from the structure parameter $C_{T}{ }^{2}$ of temperature through the following equation $[23,24]$ :

$$
C_{n}^{2}=\left(79 \times 10^{-6} \frac{\langle P\rangle}{\langle T\rangle^{2}}\right)^{2} C_{T}^{2}
$$

where $\langle P\rangle$ and $\langle T\rangle$ are the mean value of air pressure and the average temperature along the path, respectively. $C_{T}^{2}$ can be calculated directly from temperature measurements in two points separated by a particular distance [5], and is related to the $2 / 3$ power law of temperature variation along the path. $C_{T}^{2}$ can be derived from the general definition of the structure function $D_{T}$ associated with the random processes given by:

$$
D_{T}=\left\langle\left(T_{1}-T_{2}\right)^{2}\right\rangle=\left\{\begin{array}{ll}
C_{T}^{2} l_{0}^{-4 / 3} L_{p}^{2} & \text { for } 0<L_{p}<l_{0} \\
C_{T}^{2} L_{p}^{2 / 3} & \text { for } l_{0}<L_{p}<L_{0}
\end{array},\right.
$$

where $T_{1}$ and $T_{2}$ are the temperatures at two points separated by the propagation distance $L_{p}$. The terms $l_{0}$ and $L_{0}$ are the inner and outer scale of turbulence, respectively, i.e., the minimum and maximum size of air inhomogeneity where turbulence can be characterized by Kolmogorov's classical theory.

In this study, the behavior of an outdoor FSO link (with a typical path length ranging from less than $100 \mathrm{~m}$ to few $\mathrm{km}$ ) is assessed experimentally using a dedicated indoor optical chamber with a short 
optical path (about $650 \mathrm{~mm}$ ). The indoor testbed needs to be scaled in order to ensure the measurements carried out reflect that of an actual FSO link in an outdoor environment. Note that, the scintillation index $\sigma^{2}$ is dependent on $C_{n}^{2}$ and the temperature gradient. Assuming a constant $\mathrm{Cn}^{2}$ over short propagation spans of $\Delta L_{\text {indoor }}$ and $\Delta L_{\text {outdoor }}$ for indoor and outdoor FSO links, respectively, the relation between indoor experimental and outdoor links [25] can be derived as:

$$
R_{\text {in } / \text { out }}=\frac{\sigma_{\mathrm{i}_{\text {outdoor }}}^{2}}{\sigma_{\mathrm{i}_{\text {indoor }}}^{2}}=\frac{C_{n_{\text {ouddor }}}^{2}}{C_{n_{\text {indoor }}}^{2}} \times\left(\frac{\Delta L_{\text {outdoor }}}{\Delta L_{\text {indoor }}}\right)^{11 / 6} .
$$

The values of $C_{n}^{2}$ outdoor measured using the indoor chamber are between $4.5 \times 10^{-14}$ and $9.5 \times 10^{-11} \mathrm{~m}^{-2 / 3}$. Therefore, the corresponding scintillation index $\sigma$ determined is well below 0.3 , which is the upper bound for the weak turbulence regime where Eq. 2 holds. Note that, for $\sigma>0.3 \mathrm{Eq} .2$ no longer holds since the turbulence is in the saturation regime and therefore $\sigma$ is no longer proportional to $C_{n}^{2}$ [26]. Here we have adopted Eq. 5 to scale the measured $\sigma_{l}$ for the indoor environment to the outdoor condition. Since $R_{\text {in/out }}$ should be unity and assuming a typical range for $C_{n^{2}}^{2}$ outdoor between $10^{-16}$ and $10^{-15} \mathrm{~m}^{-2 / 3}$, the corresponding outdoor path length will be up to about $1 \mathrm{~km}$. Notice that, the proposed atmospheric chamber is equivalent to the typical cell size of a $5 \mathrm{G}$ wireless network. By generating higher temperature gradients in an indoor environment the same performance as that of an outdoor FSO linkis achieved. However, this ratio should be contrasted with other effects such as multiple scattering at the FSO transceivers in order to scale properly the laboratory results.

\section{EXPERIMENTAL SETUP}

This workconsiderably extends our previously published results, which were focused on the performance evaluation and assessment of an FSO link under sandstorm [12] or turbulence conditions [16]. To fully understand the effects of smog/dust and aerosols on the FSO link performance an extensive experimental measurement campaign under a controlled environment was carried out to obtain the novel statistics of the received optical power and signal characteristics at two different wavelengths.

A block diagram of the experimental setup adopted for this work is depicted in Fig. 2. Two laser sources (Global laser TX Beta) at $\lambda$ of 670 $\mathrm{nm}$ and $830 \mathrm{~nm}$ with a $50 \mathrm{MHz}$ electrical bandwidth and a $10 \mathrm{~mW}$ optical power were used. A pseudo-random bit sequence (PRBS) of length $2^{7}-1$ in the on-off keying - non-return to zero (OOK-NRZ) signal format at a data rate of $20 \mathrm{Mbps}$ was used for intensity modulation of laser sources. The modulated laser beams were then launched into the dedicated dust chamber. Two optical receivers (Rx, Thorlabs PDA10A) composed of photodetectors and trans-impedance amplifiers were used to detect distorted optical waveforms. The regenerated electrical signals at the output of the Rx were captured using a digital oscilloscope (Agilent DS09254A) for post-signal processing to obtain the $Q$-factor, eye-diagrams, and the bit error rate (BER) of the link under test and for further evaluations. All the key system parameters are summarized in Table I.

Smog/dust particles were injected into the chamber and a series of fans blowing in different directions were used to ensure homogenous distribution of particles. Indeed, keeping the particles airborne for more than one minute was a challenge; therefore, the experiment was carried out a number of times to ensure repeatability of the data generated. As for generating turbulence, it is necessary to create a temperature gradient within the chamber, which is $>6^{\circ} \mathrm{C} / \mathrm{m}$. This was achieved by blowing hot and cold air into the chamber. The fan used in the atmospheric chamber allowed us to reach an airflow up to $3 \mathrm{~m} / \mathrm{s}$. The temperature gradient was measured using 10 temperature sensors positioned equidistantly along the chamber, which were controlled using a microprocessor control unit that transmitted the information to the central signal processing computer. The temperatures were measured every second within a range of $-55^{\circ} \mathrm{C}$ to $+125^{\circ} \mathrm{C}$ with a resolution of $0.1^{\circ} \mathrm{C}$. A number of fans were used to inject both hot and cold air into the chamber. The strength of the turbulence was controlled by simply adjusting the temperature and air flow from the fan heaters and their position, thus ensuring a minimum temperature gradient $>6^{\circ} \mathrm{C} / \mathrm{m}$ along the chamber. In this work a temperature range of $20^{\circ} \mathrm{C}$ to $60^{\circ} \mathrm{C}$ was considered. This measurement set-up allowed us to readily assess the link performance under the influence of turbulence and dust particles in a controlled environment, where repeated measurement could be carried out. A picture of the experimental testbed is depicted in Fig. 3(a), whereas Fig. 3(b) shows the inside of the test chamber.

\section{FSO test chamber}

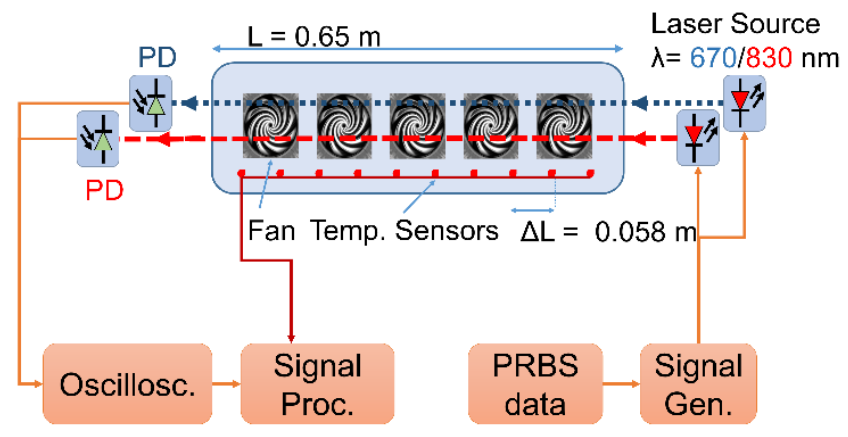

Fig. 2. Schematic block diagram of the experimental setup.

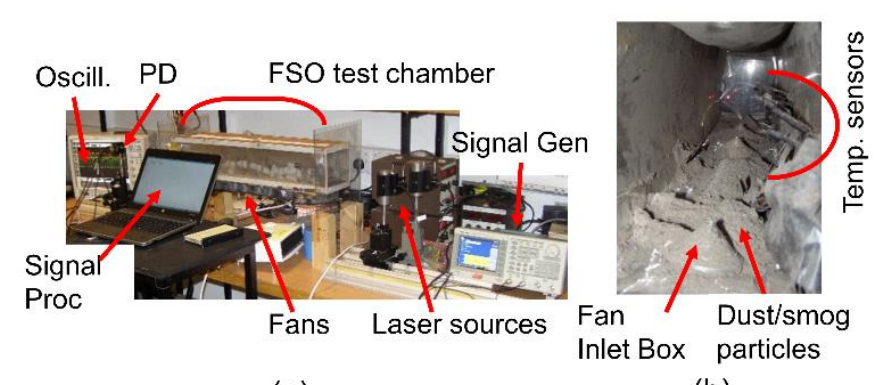

(a)

(b)

Fig. 3. Snapshot of the: (a) experimental setup, and (b) inside the test chamber.

Table I. System parameters.

\begin{tabular}{ccc}
\hline Parameter & & Value \\
\hline Input signal & Data rate & $20 \mathrm{Mbps}$ \\
(OOK-NRZ) & Wavelength $-\lambda$ & $670 \& 830 \mathrm{~nm}$ \\
Laser source & Power $-P_{\mathrm{T}}$ & $10 \mathrm{~mW}$ \\
(Global laser & Electrical bandwidth $-B_{\mathrm{TX}}$ & $50 \mathrm{MHz}$ \\
TX Beta) & Responsivity $R$ & $0.44 \mathrm{~A} / \mathrm{W}$ \\
\hline Optical & Electrical bandwidth $-B_{\mathrm{RX}}$ & $150 \mathrm{MHz}$ \\
receiver & Active area & $0.8 \mathrm{~mm}{ }^{2}$ \\
(Thorlabs & TIA gain & $5 \cdot 103 \mathrm{~V} / \mathrm{A}$ \\
PDA10A) & Dimension & $0.65 \mathrm{~m} \times 0.1 \mathrm{~m} \times 0.1 \mathrm{~m}$ \\
\hline Atmospheric & Thermal sensor spacing & $0.058 \mathrm{~m}$ \\
channel &
\end{tabular}




\section{RESULTS}

In order to analyse the effect of smog particles on FSO link performance, two samples of natural smog particles were used: (i) ash - which was collected from a common wildfire of the Central European wood; and (ii) sand - which is commonly found in urban and suburban areas particularly during highly polluted and windy days. To compare the results with the analytical approach, the particle size distribution was estimated using the magnified pictures of measured samples. The size of particles was determined from images captured using an electron microscope, see Fig. 4. The images were post-processed using Matlab to derive the probability density function (pdf) of the particle size for each sample. Because of the resolution of electron microscope used it was not possible to measure the particle size smaller than approximately $5 \mu \mathrm{m}$. The measured particle size distributions for the sand and ash can be represented via the log-normal model as depicted on processed distributions from measured data in Fig. 5(a) and 5(b), respectively. The density parameters of measured samples together with mean and standard deviations of determined log-normal distributions are summarized in Table II.

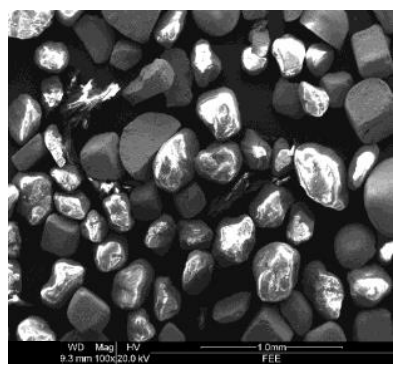

(a)

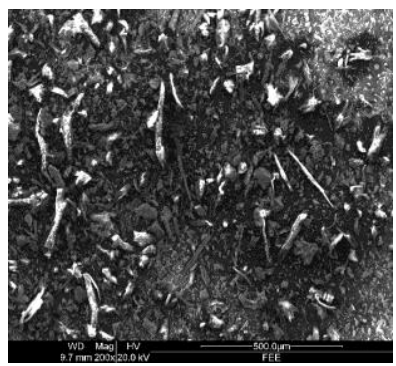

(b)
Fig. 4. Electron microscope images of: (a) sand, and (b) ash particles.

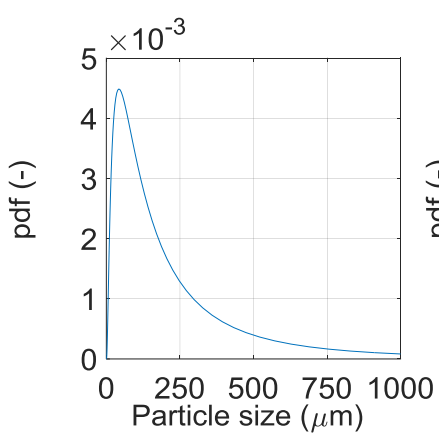

(a)

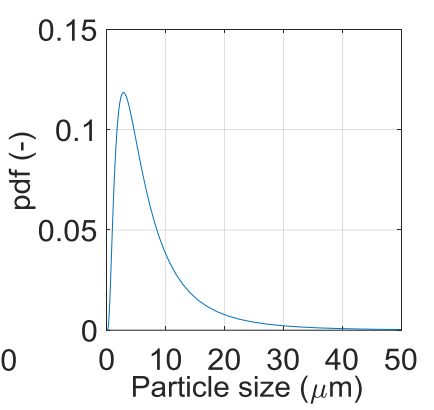

(b)
Fig. 5. Particle size distributions of: (a) sand and (b) ash sample.

Table II. Aerosol sample main characteristics and particle distribution lognormal model parameters.

\begin{tabular}{ccccc}
\hline & \multicolumn{2}{c}{$\begin{array}{c}\text { Measured sample } \\
\text { parameter }\end{array}$} & \multicolumn{2}{c}{ Log-normal model } \\
& $\begin{array}{c}\text { Weight } \\
(\mathrm{kg})\end{array}$ & $\begin{array}{c}\text { Density } \\
\left(\mathrm{kg} / \mathrm{m}^{-3}\right)\end{array}$ & Mean & $\begin{array}{c}\text { Standard } \\
\text { deviation }\end{array}$ \\
\hline Sand & 0.35400 & 1600 & 5 & 1.11447 \\
\hline Ash & 0.10054 & 750 & 1.74164 & 0.83505 \\
\hline
\end{tabular}

The FSO link performance was tested for $\lambda$ of $670 \mathrm{~nm}$ and $830 \mathrm{~nm}$ under both ash and dust conditions. Fig. 6 illustrates the extinction efficiency factor $Q_{\text {ext }}$ obtained from (1) for each sample as a function of the size parameter $x=2 \pi r / \lambda$ at $\lambda$ of 670 and $830 \mathrm{~nm}$ for sand and ash particles. This figure follows Fig. 1 where for the region $r / \lambda \ll 1 Q_{e x t}$ increases to almost 5 , and for $r / \lambda \gg>1$ it converges to 2 as expected. This indicates that the aerosol particle size and their concentration determine the energy loss in the FSO link under sand and ash environments. With reference to Fig. 6 the means of particle size distributions for sand and ash are about $50 \mu \mathrm{m}$ and $3 \mu \mathrm{m}$, respectively, therefore the FSO link under evaluation is best characterized for $r / \lambda>>1$.

Next, the eye diagrams were measured by transmitting the OOK-NRZ signal format under clear and dusty channel conditions at $\lambda$ of $830 \mathrm{~nm}$, air flow of $2 \mathrm{~m} / \mathrm{s}$ and $C_{n}^{2}=10^{-14} \mathrm{~m}^{-2 / 3}$, as shown in Fig. 7(a) and 7(b), respectively. As can be seen the eye-opening is much reduced under dust condition, which translates into a reduction in the $Q$-factor from 10 (clear channel without aerosols) to 4.5 .

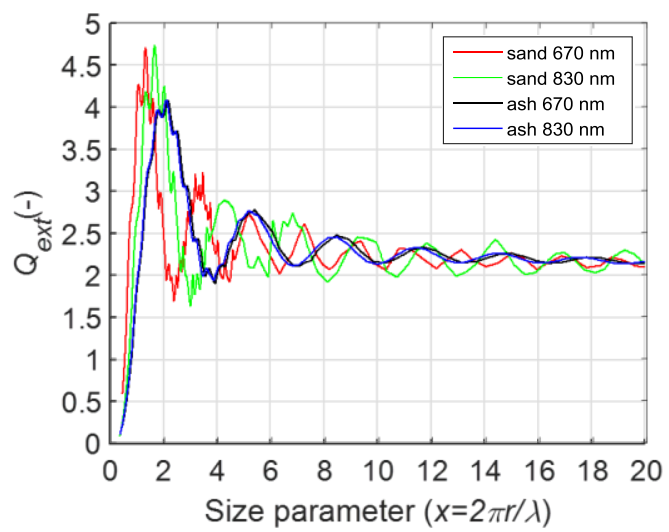

Fig. 6. Extinction efficiency against the particle size parameter for sand and ash at $\lambda$ of 670 and $830 \mathrm{~nm}$.

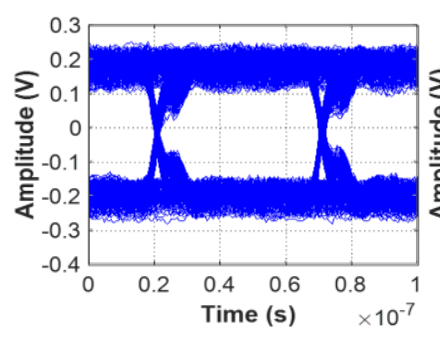

(a)

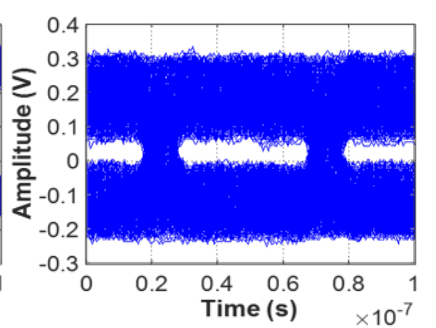

(b)
Fig. 7. Eye diagrams: (a) clear, and (b) dusty conditions for $2 \mathrm{~m} / \mathrm{s}$ air flow and $C_{n}^{2}=10^{-14} \mathrm{~m}^{-2 / 3}$.

Fig. 8 depicts the measured attenuation and the $Q$-factor as a function of the air flow with sand and ash particles for two values of $C_{n}^{2}$ of (i.e., 7.5 $\times 10^{-11} \mathrm{~m}^{-2 / 3}$ and $4.5 \times 10^{-14} \mathrm{~m}^{-2 / 3}$ ). The received signal was averaged over one minute to ensure a longer airborne time for particles within the chamber. The initial attenuation measurements under the no air flow condition (only at $0 \mathrm{~m} / \mathrm{s}$ ) were used as a reference (first value equal to $0 \mathrm{~dB}$ of attenuation) to calculate the attenuation levels, see Fig. 8 (a) and (c). As shown in Fig. 8 (a) and 8(c), the attenuation increases with $C_{n}^{2}$ for ash and sand particles at both wavelengths. In the case of ash (Fig. 8 (a)), due to the small particle size, the attenuation increases with the air flow velocity. For example, for the air flow of $2.5 \mathrm{~m} / \mathrm{s}$ the FSO attenuation is > $1 \mathrm{~dB}$ for all cases. Sand particles with larger sizes induce higher attenuations under turbulence and air flow as depicted in Fig. 8(c). For example, at an air flow of $2 \mathrm{~m} / \mathrm{s}$ for $\lambda$ of 670 the attenuation has increased from $0.1 \mathrm{~dB}$ (no turbulence) to over $1.5 \mathrm{~dB}$ with turbulence. 


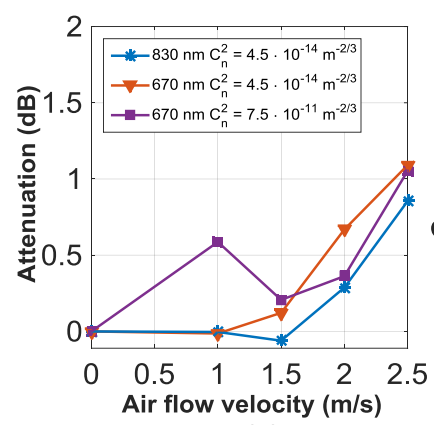

(a)

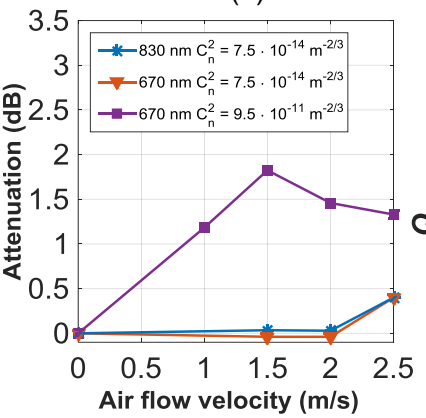

(c)

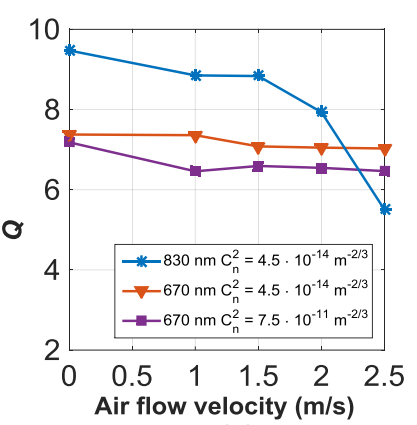

(b)

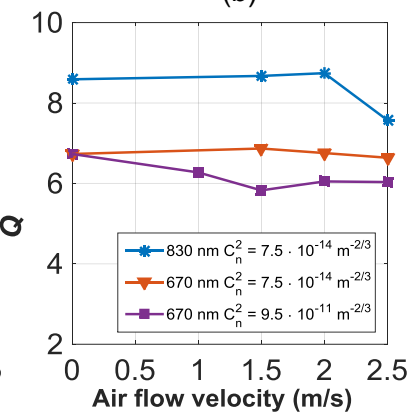

(d)
Fig. 8. (a), (c) Attenuation and (b), (d) $Q$-factor dependence on air flow velocity in turbulent channel in case of ash (top graphs) and sand (bottom graphs), respectively for each $C_{n}^{2}$ scenario.

The FSO link performance in terms of the $Q$-factor obtained from the experimental measurements is shown in Fig. 8(b) and 8(d), for ash and sand particles, respectively. In both cases, with an air flow of $2 \mathrm{~m} / \mathrm{s}$, the $Q$-factor is almost 3.5 higher at the $\lambda$ of $830 \mathrm{~nm}$ than at $670 \mathrm{~nm}$. However, for $\lambda$ of $670 \mathrm{~nm}$ the FSO link is less affected by the increase in airflow as compared to $830 \mathrm{~nm}$. The $Q$-factor reduces with turbulence. Under similar turbulence conditions, the $Q$-factor of ash particles is higher than the one of sand particles. This is due to the smaller size of ash particles, which results in reduced probability of beam blockage as compared to sand particles. However, with sand particles the FSO link is more vulnerable when under stronger turbulence conditions.

\section{CONCLUSIONS}

We have developed an experimental test bed to determine the combined effect of urban aerosols and turbulence on the FSO communications link. The experimental results demonstrated that the particle size of the aerosols clearly affects the FSO link performance. For the case of joint turbulence and scattering effects, we did not observe wave distributions as predicted theoretically, this is because the planar description of waveform is no longer valid. We also showed that under high air-flow conditions and with sand particles the FSO link experienced higher attenuation, thus leading to reduced $Q$-factor compared with ash particles. However, the link performance was mostly stable with sand particles except at very high airflow velocities. The measurements confirmed that particles pf relative large size such as sand lead to reduced performance of the FSO link under turbulent conditions, whereas smaller particles such as ash only lead to the scattering of the propagating light beam under moderate to high turbulence regimes.

Funding Information. Research of J. Libich was supported by the European social fund within the framework of the project CZ.1.07/2.3.00/30.0034.J Perez's work was partially funded by Spanish MINECO Juan de la Cierva Fellowship JCI-2012-14805. The joint research was supported by the EU COST ICT Action IC 1101 and by the Grant Agency of the CTU in Prague, grant no.SGS14/190/OHK3/3T/13.

\section{References}

1. M. A. Khalighi and M. Uysal, "Survey on Free Space Optical Communication: A Communication Theory Perspective," IEEE Commun. Surv. Tutorials 16, 2231-2258 (2014).

2. M. Uysal, C. Capsoni, Z. Ghassemlooy, A. Boucouvalas, and E. Udvary, Optical Wireless Communications, Signals and Communication Technology (Springer International Publishing, 2016).

3. C.-X. Wang, F. Haider, X. Gao, X.-H. You, Y. Yang, D. Yuan, H. Aggoune, H. Haas, S. Fletcher, and E. Hepsaydir, "Cellular architecture and key technologies for $5 \mathrm{G}$ wireless communication networks," IEEE Commun. Mag. 52, 122-130 (2014).

4. G. Parca, A. Shahpari, V. Carrozzo, G. Tosi Beleffi, and A. J. Teixeira, "Optical wireless transmission at 1.6-Tbit/s (16×100 Gbit/s) for nextgeneration convergent urban infrastructures," Opt. Eng. 52, 116102 (2013).

5. L. C. Andrews and R. L. Phillips, Laser Beam Propagation through Random Media, 2nd ed. (SPIE, 2005).

6. Z.Ghaseemlooy, W. Poopola, and S. Rajbhandri, "Optical wireless communications, system and channel modelling with matlab," CRC Press. London, UK (2012).

7. A. K. Majumdar, J. C. Ricklin, E. Leitgeb, M. Gebhart, and U. Birnbacher, "Optical networks, last mile access and applications," in Free-Space Laser Communications, Optical and Fiber Communications Reports (Springer New York, 2008), Vol. 2, pp. 273-302.

8. D. Kedar and S. Arnon, "Urban optical wireless communication networks: the main challenges and possible solutions," IEEE Commun. Mag. 42, s2-s7 (2004).

9. M. S. Awan, L. C. Horwath, S. S. Muhammad, E. Leitgeb, F. Nadeem, and M. S. Khan, "Characterization of Fog and Snow Attenuations for FreeSpace Optical Propagation," J. Commun. Vol 4, No 8 Spec. Issue Opt. Wirel. Commun. (2009).

10. "Environment and Climate Change Canada - Air - Canadian Smog Science Assessment Highlights and Key Messages," (2012).

11. M. Chin, R. A. Kahn, L. A. Remer, H. Yu, D. Rind, G. Feingold, P. K. Quinn, S. E. Schwartz, D. G. Streets, P. DeCola, and R. Halthore, "Atmospheric Aerosol Properties and Climate Impacts," (2009).

12. Z. Ghassemlooy, J. Perez, and E. Leitgeb, "On the performance of FSO communications links under sandstorm conditions," in Proceedings of the 12th International Conference on Telecommunications, ConTEL 2013 (2013), pp. 53-58.

13. M. ljaz, Z. Ghassemlooy, H. Le Minh, S. Rajbhandari, J. Perez, and A. Gholami, "Bit error rate measurement of free space optical communication links under laboratory-controlled fog conditions," in Networks and Optical Communications (NOC), 2011 16th European Conference on (IEEE, 2011), pp. 52-55.

14. S. Nauerth, F. Moll, M. Rau, C. Fuchs, J. Horwath, S. Frick, and H. Weinfurter, "Air-to-ground quantum communication," Nat. Photonics 7, 382-386 (2013).

15. E. N. T. K. Oanh, Integrated Air Quality Management: Asian Case Studies (CRC Press, 2012).

16. J. Perez, S. Zvanovec, Z. Ghassemlooy, and W. O. Popoola, "Experimental characterization and mitigation of turbulence induced signal fades within an ad hoc FSO network," Opt. Express 22, 3208-3218 (2014).

17. I. I. Kim, B. McArthur, and E. J. Korevaar, "Comparison of laser beam propagation at $785 \mathrm{~nm}$ and $1550 \mathrm{~nm}$ in fog and haze for optical wireless communications," in SPIE Proceedings (SPIE, 2001), Vol. 4214, pp. 2637.

18. M. Esmail, H. Fathallah, and M.-S. Alouini, "An Experimental Study of FSO Link Performance in Desert Environment," IEEE Commun. Lett. 20, 1-1 (2016).

19. H. C. Van de Hulst, Light Scattering by Small Particles (Dover, 1981).

20. D. R. Longtin, E. P. Shettle, J. R. Hummel, and J. D. Pryce, A Wind Dependent Desert Aerosol Model: Radiative Properties (1988).

21. R. N. Gibson, R. J. A. Atkinson, and J. D. M. Gordon, "Inherent optical 
properties of non-spherical marine-like particles from theory to observation," Oceanogr. Mar. Biol. an Annu. Rev. 45, 1-38 (2007).

22. L. E. Paramonov, "On optical equivalence of the randomly oriented ellipsoidal and polydisperse spherical particles. Extinction, scattering and absorption cross sections," in Satellite Remote Sensing, D. K. Lynch, ed. (SPIE, 1994), pp. 358-360.

23. S. Corrsin, "On the Spectrum of Isotropic Temperature Fluctuations in an Isotropic Turbulence," J. Appl. Phys. 22, 469 (1951).

24. A. M. Obukhov, "Structure of the temperature field in turbulent flow,"
Izv. Akad. Nauk. SSSR 19, 58-59 (1949).

25. Z. Ghassemlooy, H. Le Minh, S. Rajbhandari, J. Perez, and M. Ijaz, "Performance Analysis of Ethernet/Fast-Ethernet Free Space Optical Communications in a Controlled Weak Turbulence Condition," J. Light. Technol. 30, 2188-2194 (2012).

26. S. F. Clifford, G. R. Ochs, and R. S. Lawrence, "Saturation of optical scintillation by strong turbulence," J. Opt. Soc. Am. 64, 148 (1974).

For the online copyrighted and edited version please check:

J. Libich, J. Perez, S. Zvanovec, Z. Ghassemlooy, R. Nebuloni, and C. Capsoni, "Combined effect of turbulence and aerosol on freespace optical links," Appl. Opt. 56, 336-341 (2017). https://doi.org/10.1364/AO.56.000336

(c) 2017 Optical Society of America.]. One print or electronic copy may be made for personal use only. Systematic reproduction and distribution, duplication of any material in this paper for a fee or for commercial purposes, or modifications of the content of this paper are prohibited. 